\title{
Perpendicular spin valves with ultrathin ferromagnetic layers: Magnetoelectronic circuit investigation of finite-size effects
}

\author{
Alexey A. Kovalev, ${ }^{1}$ Gerrit E. W. Bauer, ${ }^{1}$ and Arne Brataas ${ }^{2}$ \\ ${ }^{1}$ Kavli Institute of NanoScience, Delft University of Technology, 2628 CJ Delft, The Netherlands \\ ${ }^{2}$ Department of Physics, Norwegian University of Science and Technology, N-7491 Trondheim, Norway \\ (Received 27 April 2005; revised manuscript received 29 November 2005; published 3 February 2006)
}

\begin{abstract}
We address two finite-size effects in perpendicular transport through multilayers of ferromagnetic and normal metal layers: (i) the transport properties depend on the magnetic layer thickness when of the order or thinner than the spin-flip diffusion length and (ii) magnetic layers with thickness approaching the magnetic coherence length become transparent for spin currents polarized perpendicular to the magnetization. We use magnetoelectronic circuit theory to investigate both effects on angular magnetoresistance (aMR) and spintransfer torque in perpendicular spin valves. We analyze recent aMR experiments to determine the spin-flip diffusion length in the ferromagnet permalloy as well as the Py|Co interface spin-mixing conductance and propose a method to measure the ferromagnetic coherence length.
\end{abstract}

DOI: 10.1103/PhysRevB.73.054407

PACS number(s): 75.70.Ak, 72.25.Ba, 75.60.Jk, 72.25.Rb

\section{INTRODUCTION}

Since the discovery of the giant magnetoresistance (GMR), ${ }^{1}$ electron transport in magnetic metallic heterostructures has been studied intensively and with considerable progress. The field developed from studies of large area multilayers of ferromagnetic $(F)$ and normal metals $(N)$ in which the current flows in the plane of the interfaces (CIP) to nanostructures with current perpendicular to the planes (CPP). ${ }^{2}$ Current-induced magnetization excitation has been predicted for perpendicular $F|N| F$ spin valves ${ }^{3,4}$ and subsequently observed. ${ }^{5-8}$ In these experiments applied currents excite a spin accumulation in the normal metal spacer that exerts a torque on the ferromagnets. When this torque overcomes the damping, the magnetization starts to precess coherently, possibly leading to a complete magnetization reversal. ${ }^{9}$ By fits of the parameters of the diffusion equation ${ }^{10}$ to a wealth of experimental data of the GMR in CPP structure, the spindependent interface and bulk material resistances of the most important transition metal combinations are well known by now. ${ }^{2,11}$ First-principles calculations in general agree well with the experimental values. ${ }^{12}$ Also in view of possible applications for switching purposes in magnetic random access memories, a comparably accurate modeling of the spin torque as a function of material combinations and applied bias is desirable.

Physically, the spin-transfer torque is a consequence of angular momentum conservation when a spin current polarized transverse to the magnetization direction is absorbed at the magnetic interface. ${ }^{13}$ The transverse spin current can penetrate the ferromagnet up to a skin depth equal to the ferromagnetic coherence length $\lambda_{c}=\pi /\left|k_{\uparrow}^{F}-k_{\downarrow}^{F}\right|$. In transition metals $\lambda_{c}$ is much smaller than all other length scales such as spin-diffusion length or mean-free path. ${ }^{14-16}$ When the ferromagnetic layer thickness $d_{F} \gg \lambda_{c}$ the spin-transfer torque is a pure interface property governed by the so-called spinmixing conductance, ${ }^{17}$ which is accessible to first-principles calculations. $^{18}$

An excellent method to measure the torque and mixing conductance is the normalized angular magnetoresistance
(aMR) of perpendicular $F|N| F$ spin valves ${ }^{19-21}$

$$
\operatorname{aMR}(\theta)=\frac{R(\theta)-R(0)}{R(\pi)-R(0)},
$$

where $R(\theta)$ is electric resistance when the two magnetizations are rotated by an angle $\theta$ with respect to each other. Deviations of the aMR as a function of $\cos \theta$ from a straight line are proof of a finite mixing conductance. ${ }^{22}$ Systematic new measurements of the aMR have been carried out recently by Urazhdin et $a l^{23}$ on permalloy (Py) $\mid \mathrm{Cu}$ spin valves as a function of the Py thicknesses.

Interesting effects such as nonmonotonic aMR, change of sign of the spin-transfer torque, and strongly reduced critical currents for magnetization reversal have been predicted for asymmetric spin valves. ${ }^{24-26}$ Asymmetry here means that the two ferromagnets in the spin valve are not equivalent for spin transport. This can be achieved by different thicknesses of the magnetically active regions of otherwise identical ferromagnetic contacts, but only when the spin-flip diffusion length in the ferromagnet $l_{s d}^{F}$ is of the order or larger than one of the magnetic layer thicknesses. The magnetically soft Py is the material of choice, but its spin-flip diffusion length is only $l_{s d}^{F} \cong 5 \mathrm{~nm} .{ }^{11}$ Urazhdin et al. ${ }^{23}$ investigated spin valves with ultrathin $d_{F} \leq l_{s d}^{F}$, which means that the analysis of these experiments requires solution of the spin and charge diffusion equation in the ferromagnet.

Detailed calculations for transition metals ${ }^{16,27}$ confirm that a transverse spin current can penetrate the ferromagnet over distances $\leqslant 1 \mathrm{~nm}$ as a consequence of incomplete destructive quantum interference. Urazhdin et al. investigated spin valves with Py layers of such thicknesses, claiming to observe an effect of this transverse component on the aMR. In weak ferromagnets like CuNi or PdNi alloys in which $\lambda_{c}$ may become larger than the scattering mean-free path, the transverse component of spin current and accumulation can be treated semiclassically. ${ }^{28}$ It is shown below that an effective conductance parameter ("mixing transmission") can be introduced to parametrize transport in both regimes. 
In this paper we treat the size effects related to $d_{F} \leqslant l_{s d}^{F}$ (Sec. II) and $d_{F} \lesssim \lambda_{c}$ (Sec. III) (but $\lambda_{c}$ much smaller than the spin diffusion length). In Sec. II we apply magnetoelectronic circuit theory ${ }^{17}$ combined with the diffusion equation to the $F|N| F \mid N$ spin valves studied by Urazhdin et al. We demonstrate that the angular magnetoresistance provides a direct measure for the mixing conductance ${ }^{22}$ and find that the nonmonotonicity in the aMR is indeed caused by the asymmetry as predicted. For $F|N| F|N| F$ structures, that are also of interest because of their increased spin torque, ${ }^{29,30}$ we obtain several analytical results. The approach from Sec. II is generalized in Sec. III, allowing us to treat ultrathin ferromagnetic layers or weak ferromagnets. ${ }^{31,32,27}$ We find that there should be no measurable effects of $\lambda_{c}$ on the aMR in $F|N| F \mid N$ structures, but predict that the torque acting on the thin layer is modified. We proceed to conclude that the coherence length should be observable in the aMR of $F|N| F|N| F$ structures. Finally, we propose a setup to measure the ferromagnetic coherence length in a three-terminal device.

\section{MAGNETOELECTRONIC CIRCUIT THEORY AND DIFFUSION EQUATION FOR SPIN VALVES}

In this section we assume that $\lambda_{c} \ll d_{F}$. In Sec. II A we recapitulate some old results: the magnetoelectronic circuit theory for spin valves, with emphasis on the inclusion of the spin-flip diffusion in the ferromagnetic layers when the ferromagnetic layer thickness $d_{F}$ is of the same order as the spin-flip diffusion length in the ferromagnet $l_{s d}^{F}$. In Sec. II B we apply these results to recent experiments by Urazhdin et $a l$. in which we can disregard spin-flip in the $\mathrm{Cu}$ spacers. In Sec. II C we present results for symmetric $F|N| F|N| F$ structures.

\section{A. Magnetoelectronic circuit theory and diffusion equation}

Magnetoelectronic circuit theory ${ }^{14}$ has been designed to describe charge and spin transport in disordered or chaotic multiterminal ferromagnet-normal metal hybrid systems with noncollinear magnetizations. The material parameters of the theory are the bulk and interface spin-dependent conductances, as well a the so-called interface spin-mixing conductance $G_{\uparrow \downarrow}$. For spin valves, circuit theory can be shown to be equivalent to a diffusion equation when $\operatorname{Im} G_{\uparrow \downarrow} \approx 0$, which is usually the case for intermetallic interfaces. ${ }^{24}$ When the thickness of the ferromagnetic metal layer $d \gg l_{s d}^{F}$, the layer bulk resistance can be effectively replaced by that of a magnetically active region close to the interface of thickness $l_{s d}^{F}$. When connected to a reservoir or other type of spin sink, the effective thickness becomes $l_{s d}^{F} \tanh \left(d_{F} / l_{s d}^{F}\right) \cdot{ }^{24}$

The aMR for general $N|F| N|F| N$ structures with $\operatorname{Im} G_{\uparrow \downarrow}=0$ as derived previously ${ }^{24}$ reads

$$
\mathrm{R}(\theta)=R_{\uparrow \downarrow}+R_{1}+R_{2}-\frac{R_{\uparrow \downarrow}\left(R_{1-}+\alpha R_{2-}\right)^{2}+\left(1-\alpha^{2}\right)\left[R_{1-}^{2} R_{2}+R_{2-}^{2}\left(R_{1}+R_{\uparrow \downarrow}\right)\right]}{\left(R_{\uparrow \downarrow}+R_{1}\right)\left(R_{\uparrow \downarrow}+R_{2}\right)-\alpha^{2} R_{1} R_{2}},
$$

with $\quad \alpha=\cos \theta, \quad 4 R_{1(2)}=1 / G_{1(2) \uparrow}+1 / G_{1(2) \downarrow}-2 R_{\uparrow \downarrow}, \quad 4 R_{1(2)-}$ $=1 / G_{1(2) \uparrow}-1 / G_{1(2) \downarrow}, \quad P_{1(2)}=R_{1(2)-} / R_{1(2)}, \quad$ and $\quad 2 R_{\uparrow \downarrow}$ $=1 / G_{1 \uparrow \downarrow}+1 / G_{2 \uparrow \downarrow}$, where $G_{1(2) \uparrow}$ and $G_{1(2) \downarrow}$ are conductances of the left (right) ferromagnet including the left (right) normal layer, and $G_{1 \uparrow \downarrow}$ and $G_{2 \uparrow \downarrow}$ are mixing conductances of the middle normal metal with adjacent ferromagnet interfaces as shown in Fig. 1. The torques felt by the first and second ferromagnetic layers become

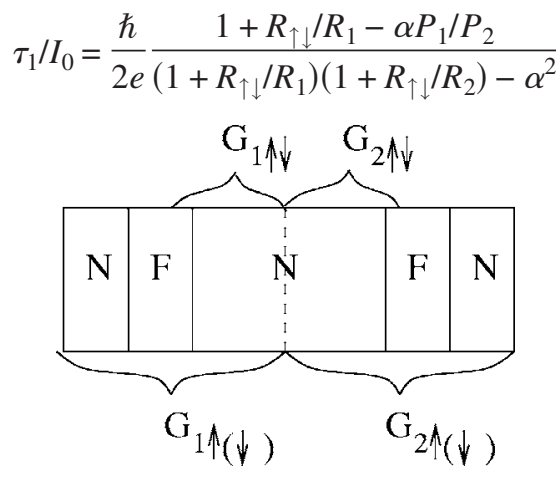

FIG. 1. Definition of conductances $G_{1(2) \uparrow(\downarrow)}$ and mixing conductances $G_{1(2) \uparrow \downarrow}$ for $N|F| N|F| N$ structure.

$$
\tau_{2} / I_{0}=\frac{\hbar}{2 e} \frac{1+R_{\uparrow \downarrow} / R_{2}-\alpha P_{2} / P_{1}}{\left(1+R_{\uparrow \downarrow} / R_{1}\right)\left(1+R_{\uparrow \downarrow} / R_{2}\right)-\alpha^{2}} .
$$

When we approximate the mixing conductance $1 / R_{\uparrow \downarrow}$ by the Sharvin conductance of the normal metal, Eqs. (3) and (4) coincide with the expressions in Ref. 33 for asymmetric $N|F| N|F| N \quad$ spin $\quad$ valves $\quad$ with $\quad \Lambda_{L(R)}^{2} \equiv 2 R_{1(2)} / R_{\uparrow \downarrow}+1$, $P_{L(R)} \Lambda_{L(R)}^{2}=2 R_{1(2)-} / R_{\uparrow \downarrow}$.

\section{B. Extraction of the mixing conductance from experiment and asymmetric spin valves}

Most material parameters in circuit theory are those of the two-channel resistor model. They can be determined for the collinear magnetic configurations, i.e., via the (binary) GMR. The only additional parameters for the noncollinear transport are the interface mixing conductances $G_{i \uparrow \downarrow}^{r}$, assumed here to be real. These can be found from a single parameter fit of the experimental aMR or from band structure calculations. A symmetric $F|N| F$ structure is most suitable to carry out this program. The thus obtained $G_{i \uparrow \downarrow}^{r}$ should be transferable to other (asymmetric) structures grown by equivalent techniques. Urazhdin et al. fitted their experimental results for 


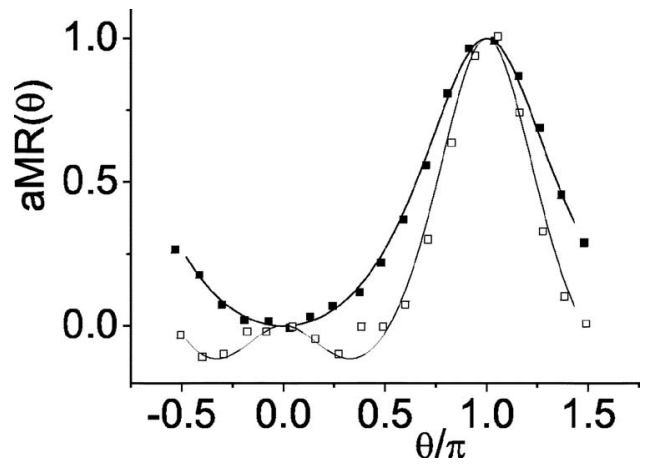

FIG. 2. aMR of the $F|N| F \mid N$ structure for two thicknesses of the right ferromagnetic layer $d_{2}=0.27 l_{s d}, 2.2 l_{s d}\left(d_{1}>l_{s d}\right)$. The filled (large $d_{2}$ ) and open (small $d_{2}$ ) squares are the experimental data Ref. 23.

the normalized aMR by the simple formula ${ }^{21}$ that follows from circuit theory ${ }^{14}$

$$
\operatorname{aMR}(\theta)=\frac{1-\cos \theta}{\chi(1+\cos \theta)+2} .
$$

For symmetric junctions we identify $\chi=2 R / R_{\uparrow \downarrow}$ [see Eq. (18) in Ref. 24].

Urazhdin et al. ${ }^{23}$ used the structures $\mathrm{Nb}(150) \mathrm{Cu}(20)$ $\operatorname{FeMn}(8) \operatorname{Py}\left(d_{1}\right) \mathrm{Cu}(10) \operatorname{Py}\left(d_{2}\right) \mathrm{Cu}(20) \mathrm{Nb}(150)$, where the numbers in parentheses are the thicknesses in $\mathrm{nm}$. The exchange bias antiferromagnet FeMn is treated as a perfect spin sink, which means that the effective thickness of the left Py layer becomes $l_{s d}^{F} \tanh \left(d_{1} / l_{s d}^{F}\right)=0.8 l_{s d}\left(d_{1}=6 \mathrm{~nm}\right.$, $\left.l_{s d}^{F}=5.5 \mathrm{~nm}\right)$. Note that this device is not exactly symmetric when $d_{2} \gg l_{s d}^{F}$ as $d_{1}$ is not much larger than $l_{s d}^{F}$, but the calculated deviations from the fitted mixing resistances are smaller than the experimental error bars. When we replace $d_{1}$ by $l_{s d}^{F}$ and $d_{2} \gg l_{s d}^{F}$ the sample is symmetric and the aMR is well represented by Eq. (5) with $\chi=1.96$ (see Fig. 2). ${ }^{23}$

We can use the measured value of $\chi$ to derive the mixing conductance $1 /\left(A R_{\uparrow \downarrow}\right)$ of an interface with area $A$ by $R_{\uparrow \downarrow}=2 R / \chi$. For comparison with first-principles calculations for point contacts based on the scattering theory of transport, ${ }^{18}$ the Sharvin resistance of the normal metal should be added, ${ }^{22}$

$$
R_{\uparrow \downarrow}^{p c}=R_{\uparrow \downarrow}+R_{s h} .
$$

Using the notation

$$
\begin{gathered}
A R=l_{s d} \rho_{P y}^{*}+A R_{P y C u}^{*}-A R_{\uparrow \downarrow} / 2, \\
A R_{-}=l_{s d} \rho_{P y}^{*} \beta_{P y}+A R_{P y C u}^{*} \gamma,
\end{gathered}
$$

we may substitute the well-established material parameters for bulk and interface resistances of $\mathrm{Cu} \mid \mathrm{Py}^{34} l_{s d} \rho_{P y}^{*}$ $=1.4 \mathrm{f} \Omega \mathrm{m}^{2}, \quad l_{s d}=5.5 \mathrm{~nm}, \quad A R_{P y C u}^{*}=0.5 \mathrm{f} \Omega \mathrm{m}^{2}, \quad \beta_{P y}=0.7$, $\gamma=0.7$, disregarding the small bulk resistance of $\mathrm{Cu}$ which led us to $A R_{\uparrow \downarrow}=1.3 \mathrm{f} \Omega \mathrm{m}^{2}$ and $\eta=1.49$. This value of the mixing resistance is larger than the Sharvin resistance $A R_{s h}=1 / G=0.878 \mathrm{f} \Omega \mathrm{m}^{2}$ of $\mathrm{Cu}$ used by Xiao et al. ${ }^{33}$ The point-contact mixing resistance of the $\mathrm{Cu} / \mathrm{Py}$ interface that should be compared with band structure calculations is

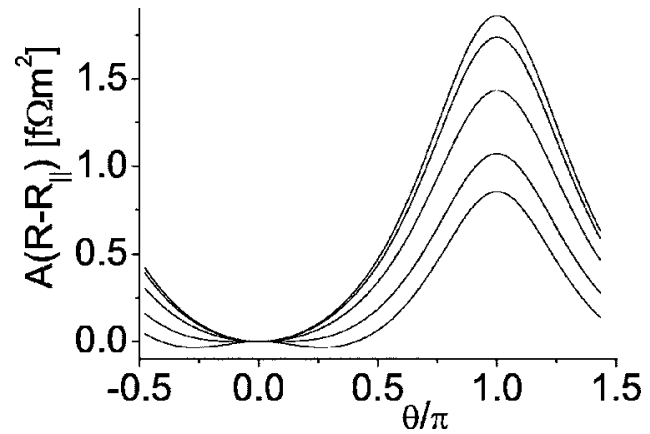

FIG. 3. Angular magnetoresistance $\mathrm{R}(\theta)-\mathrm{R}(0)$ of the $F|N| F \mid N$ structure for different thicknesses of the right ferromagnetic layer $d_{2}=0.27 l_{s d}, 0.5 l_{s d}, 2 l_{s d}, 2.5 l_{s d}$ and $\infty$ [starting from the lower curve respectively $\left.\left(d_{1} \gg l_{s d}^{F}, A R_{P y N b}=3 \mathrm{f} \Omega \mathrm{m}^{2}\right)\right]$.

$A R_{\uparrow \downarrow}^{p c .}=2.2 \mathrm{f} \Omega \mathrm{m}^{2}$, somewhat smaller than that found in Ref. $22\left(2.56 \mathrm{f} \Omega \mathrm{m}^{2}\right)$. Both results are close to the band structure calculations $^{18}$ of the point-contact mixing resistance for the disordered $\mathrm{Cu} \mid \mathrm{Co}$ interface $\left(2.4 \mathrm{f} \Omega \mathrm{m}^{2}\right)$.

In Fig. 2 we compare plots of Eq. (2) with experimental aMR curves for symmetric and asymmetric $F|N| F \mid N$ multilayers, ${ }^{35}$ identifying the following relations between parameters:

$$
\begin{gathered}
A R_{1}=l_{s d} \rho_{P y}^{*}+A R_{P y C u}^{*}-A R_{\uparrow \downarrow} / 2, \\
A R_{2}=d_{2} \rho_{P y}^{*}+A R_{P y C u}^{*}+A R_{P y N b}-A R_{\uparrow \downarrow} / 2, \\
A R_{1-}=l_{s d} \rho_{P y}^{*} \beta_{P y}+A R_{P y C u}^{*} \gamma, \\
A R_{2-}=d_{2} \rho_{P y}^{*} \beta_{P y}+2 A R_{P y C u}^{*} \gamma .
\end{gathered}
$$

We assume that the spin current into the superconductor vanishes. The resistance between the right ferromagnet and the right reservoir was taken to be $A R_{P y N b}=5 \mathrm{f} \Omega \mathrm{m}^{2}$. This is larger than the $A R_{P y N b}=3 \mathrm{f} \Omega \mathrm{m}^{2}$ reported in Ref. 34, but gives better agreement with the experiment. We observe good fits in Fig. 2, nicely reproducing the nonmonotonic behavior around zero angle.

In Fig. 3 we plot the angular magnetoresistance for different thicknesses of the right Py layer, all relative to the parallel configuration, but not normalized to a relative scale as above. The lower curve was obtained from Eq. (2), the others were calculated numerically solving the bulk layer spindiffusion equation in the ferromagnet. The nonmonotonic angular magnetoresistance disappears when the right ferromagnetic layer becomes thicker and therefore the sample more symmetric. For the set of parameters in Fig. 3 the nonmonotonic behavior is rather weak but with circuit theory we can readily propose samples that maximize the effect. The minimum of the angular magnetoresistance Eq. (2) at finite $\theta_{1}$ that coincides with a zero of the spin-transfer torque on the left ferromagnet ${ }^{24,25}$ 


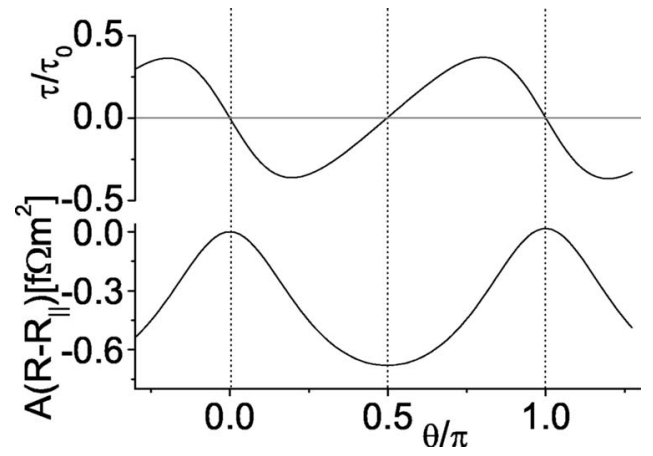

FIG. 4. Angular magnetoresistance $\mathrm{R}(\theta)-\mathrm{R}(0)$ and spin-transfer torque on the left ferromagnet for the $F|N| F \mid N$ structure with right $F$-layer thickness $d_{2}=0.27 l_{s d}\left(A R_{P y N b} \rightarrow \infty, \tau_{0}=I_{0} \hbar / 2 e\right)$.

$$
\cos \theta_{1}=\frac{\left(R_{\uparrow \downarrow}+R_{1}\right) R_{2-}}{R_{1 \_} R_{2}} .
$$

To observe the effect clearly, $\cos \theta_{1}$ should be small, which can be achieved by increasing $R_{2}$, e.g., by the resistance of the right-most normal metal (within the spin-flip diffusion length). In Fig. 4 we plot the angular magnetoresistance Eq. (2) and the spin-transfer torque on the left ferromagnet Eq. (3) when the resistance of the right contact is $A R_{P y N b} \rightarrow \infty$.

\section{Analysis of symmetric $F|N| F|N| F$ structures}

Our approach offers analytic results for symmetric $F|N| F|N| F$ structures when the outer layers are thicker than $l_{s d}^{F}$. In Fig. 5 we plot the angular magnetoresistance when the magnetizations of the outer layers are kept parallel for material parameters that are the same as above and close to setup $B$ from Ref. 23. When the middle layer thickness $d_{3} \gg l_{s d}^{F}$, the angular magnetoresistance is equal to that of two symmetric $F|N| F$ structures in series. The analytical formula for the angular magnetoresistance in the regime $d_{3} \ll l_{s d}^{F}$ is presented in the Appendix. For $d_{3} \gtrsim 0.3 l_{s d}^{F}$ we cannot disregard spin flip in the middle layer and compute the resistances numerically.

A symmetric $F|N| F|N| F$ setup with antiparallel outer layers can increase the torque. ${ }^{29}$ Enhancement by a factor of 2 was reported by Nakamura et al. ${ }^{30}$ This result can be ob-

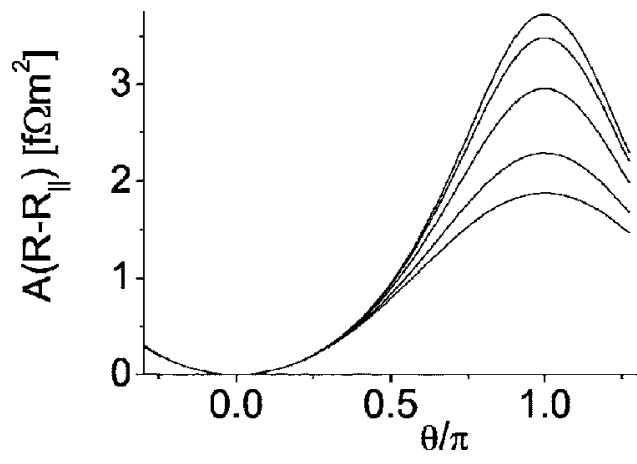

FIG. 5. Angular magnetoresistance $\mathrm{R}(\theta)-\mathrm{R}(0)$ of the $F|N| F|N| F$ structure for the middle $F$ layer thicknesses $d=0.27 l_{s d}, 0.5 l_{s d}, 2 l_{s d}, 2.5 l_{s d}$ and $\infty$ (starting from the lower curve, respectively). The parallel resistance is subtracted.

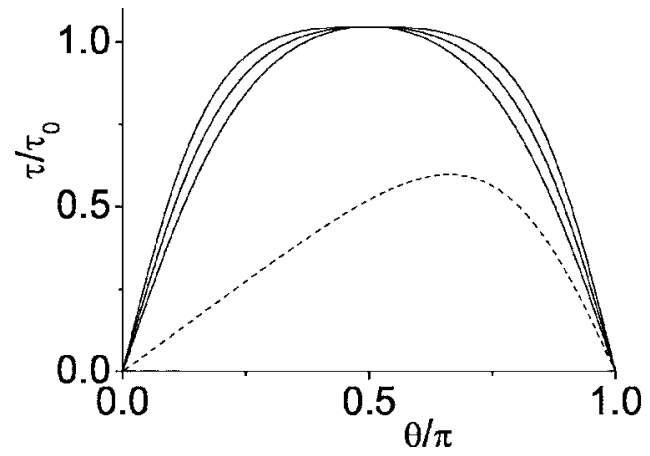

FIG. 6. The spin-transfer torque on the middle ferromagnet for the $F|N| F|N| F$ structure for the thickness of the middle layer $d=0.27 l_{s d}, 0.8 l_{s d}$ and $10 l_{s d}$ starting from the lower curve respectively (by bold line), the same for the corresponding symmetric $F|N| F$ structure (by dashed line), $\tau_{0}=I_{0} \hbar / 2 e$.

tained from the magnetoelectronic circuit theory. ${ }^{38}$ With a current bias $I_{0}$, assuming $d_{3} \ll l_{s d}^{F}$, we derived a simple formula (note the similarity with the torque on the base contact of the three-terminal spin-flip transistor ${ }^{22}$ ),

$$
\tau / I_{0}=\frac{\hbar}{2 e} \frac{2 R_{-}|\sin \theta|}{R_{\uparrow \downarrow}+R \sin ^{2} \theta},
$$

without invoking the parameters of the middle layer. When $d_{3} \gg l_{s d}$ we can divide system into two $F|N| F$ spin valves in series. Taking into account Eq. (3), the torque can be written down immediately,

$$
\begin{aligned}
\tau / I_{0} & =\tau_{F N F}(\theta) / I_{0}+\tau_{F N F}(\pi-\theta) / I_{0} \\
& =\frac{\hbar}{2 e} \frac{R_{-}|\sin \theta|}{R_{\uparrow \downarrow}+R(1+\cos \theta)}+\frac{\hbar}{2 e} \frac{R_{-}|\sin \theta|}{R_{\uparrow \downarrow}+R(1-\cos \theta)} .
\end{aligned}
$$

In Fig. 6 we plot results of these two analytic formulas as well as results of numeric calculations for the case $d=0.8 l_{s d}$. Note that these curves are symmetric with respect to $\theta=\pi / 2$. By the dashed line we plot the torque for the corresponding symmetric $F|N| F$ structure.

\section{COHERENT REGIME}

The intentions of Urazhdin et al. ${ }^{23}$ to search for coherence effects in ultrathin magnetic layers encouraged us to study the regime $d_{F} \lesssim \lambda_{c}$. In this section we formulate the magne-

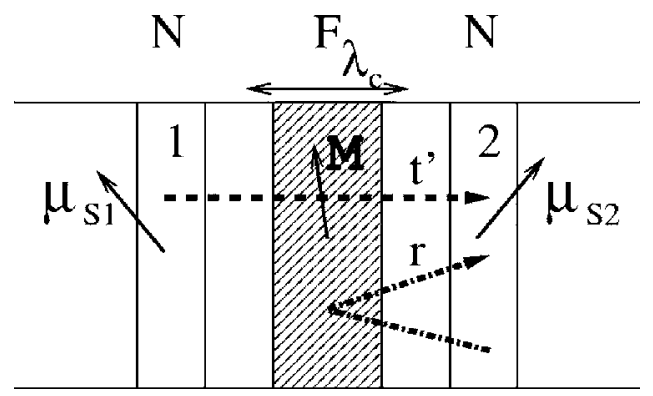

FIG. 7. A contact through a thin ferromagnet between two normal metals nodes. The current is evaluated in the node 2 . 
toelectronic circuit theory that includes coherence effects in this regime in two and three terminal multilayer structures. Since $\lambda_{c}$ is only a couple of monolayers, we are allowed to disregard spin-flip and diffuse scattering in the ferromagnetic material bulk layers.

\section{A. Extended magnetoelectronic circuit theory}

We consider an $N 1|F| N 2$ circuit element, choosing the normal metals as nodes with a possibly noncollinear spin accumulation and the entire $F$ layer including the interfaces as resistive element (see Fig. 7). This allows us to treat the ferromagnet fully quantum mechanically by scattering theory. The current through the ferromagnet depends on the potential drop between and the spin accumulation in each of the normal metal nodes. Spin $\mathbf{I}_{s}$ and charge $I_{0}$ currents can conveniently expressed as $2 \times 2$ matrices in Pauli spin space $\hat{I}=\left(\hat{1} I_{0}+\hat{\boldsymbol{\sigma}} \cdot \mathbf{I}_{s}\right) / 2$, where $\hat{\boldsymbol{\sigma}}$ is the vector or Pauli spin matrices and $\hat{1}$ the $2 \times 2$ unit matrix. On the normal metal side ${ }^{14}$ in the region 2 ,

$$
\hat{I}=\frac{e}{h}\left\{\sum_{n m}\left[\hat{t}^{\prime n m} \hat{f}^{N 1}\left(\hat{t}^{\prime n m}\right)^{\dagger}-\delta_{n m} \hat{f}^{N 2}+\hat{r}^{n m} \hat{f}^{N 2}\left(\hat{r}^{n m}\right)^{\dagger}\right]\right\},
$$

where $\hat{r}^{m n}$ is the spin-dependent reflection coefficient for electrons reflected from channel $n$ into channel $m$ in the node 2, $\hat{t}^{\prime m n}$ is the spin-dependent transmission coefficient for electrons transmitted from channel $n$ in the node 1 into channel $m$ in the node 2 and $\delta_{n m}$ is the Kronecker delta symbol.

In the absence of spin-flip processes, the matrices $\hat{r}^{m n}$ and $\hat{t}^{\prime m n}$ should be diagonal in spin space provided the axis $z$ is parallel to the magnetization of the ferromagnet (we are free to chose this frame reference as it is more convenient). Expressing the spin-dependent distribution matrices in nodes 1 and 2 via Pauli matrices; $\hat{f}^{N}=\hat{1} f_{0}^{N}+\hat{\boldsymbol{\sigma}} \mathbf{f}_{s}^{N}$ and the unit vector $\mathbf{m}_{z}$ parallel to the axis $z$ we obtain for spin and charge currents in the node $N 2$

$$
\begin{gathered}
I_{0}=\left(G_{\uparrow}+G_{\downarrow}\right) \Delta f_{0}^{N}+\left(G_{\uparrow}-G_{\downarrow}\right) \Delta \mathbf{f}_{s}^{N} \cdot \mathbf{m}_{z}, \\
\mathbf{I}_{s}=\mathbf{m}_{z}\left[\left(G_{\uparrow}-G_{\downarrow}\right) \Delta f_{0}^{N}+\left(G_{\uparrow}+G_{\downarrow}\right) \Delta \mathbf{f}_{s}^{N}\right] \\
-2\left(\mathbf{m}_{z} \times \mathbf{f}_{s}^{N 2} \times \mathbf{m}_{z}\right) \operatorname{Re} G_{\uparrow \downarrow}^{r N 2 \mid F}+2\left(\mathbf{f}_{s}^{N 2} \times \mathbf{m}_{z}\right) \operatorname{Im} G_{\uparrow \downarrow}^{r N 2 \mid F} \\
+2\left(\mathbf{m}_{z} \times \mathbf{f}_{s}^{N 1} \times \mathbf{m}_{z}\right) \operatorname{Re} G_{\uparrow \downarrow}^{t N 1 \mid N 2}-2\left(\mathbf{f}_{s}^{N 1} \times \mathbf{m}_{z}\right) \operatorname{Im} G_{\uparrow \downarrow}^{t N 1 \mid N 2},
\end{gathered}
$$

where $\Delta f_{0}^{N}=f_{0}^{N 1}-f_{0}^{N 2}$ and $\Delta \mathbf{f}_{s}^{N}=\mathbf{f}_{s}^{N 1}-\mathbf{f}_{s}^{N 2}$. This agrees with the result of Ref. 14 except for the following terms involving the mixing transmission: ${ }^{27,31,32}$

$$
\begin{gathered}
G_{\uparrow \downarrow}^{r N \mid F}=\frac{e^{2}}{h} \sum_{n m}\left[\delta_{n m}-r_{\uparrow}^{n m}\left(r_{\downarrow}^{n m}\right)^{*}\right] ; \\
G_{\uparrow \downarrow}^{t N 1 \mid N 2}=\frac{e^{2}}{h} \sum_{n m} t_{\uparrow}^{\prime n m}\left(t_{\downarrow}^{\prime n m}\right)^{*} .
\end{gathered}
$$

The torque acting on the magnetization through the interface adjacent to $N 2$ is the transverse component of the spin current flowing into the ferromagnet,

$$
\begin{aligned}
\vec{\tau}_{2}= & -2\left(\mathbf{m}_{z} \times \mathbf{f}_{s}^{N 2} \times \mathbf{m}_{z}\right) \operatorname{Re} G_{\uparrow \downarrow}^{r N 2 \mid F}+2\left(\mathbf{f}_{s}^{N 2} \times \mathbf{m}_{z}\right) \operatorname{Im} G_{\uparrow \downarrow}^{r N 2 \mid F} \\
& +2\left(\mathbf{m}_{z} \times \mathbf{f}_{s}^{N 1} \times \mathbf{m}_{z}\right) \operatorname{Re} G_{\uparrow \downarrow}^{t N 1 \mid N 2}-2\left(\mathbf{f}_{s}^{N 1} \times \mathbf{m}_{z}\right) \operatorname{Im} G_{\uparrow \downarrow}^{t N 1 \mid N 2} .
\end{aligned}
$$

When two opposite direction of the magnetization $\mathbf{M}$ and $\mathbf{- M}$ are equivalent for the transport, we obtain $G_{\uparrow \downarrow}^{t N 1 \mid N 2}=G_{\uparrow \downarrow}^{t N 2 \mid N 1}$ as a consequence of time reversibility. This condition should hold in most cases (e.g., Stoner model is isotropic in spin space). The mixing transmission describes the part of the transverse spin current that is not absorbed by the ferromagnet and vanishes when the ferromagnetic layer is thicker than the ferromagnetic coherence length $\lambda_{c} \cdot{ }^{27} \mathrm{It}$ is complex, its modulus representing the transmission probability and the phase of the rotation of the transverse spin current by the ferromagnetic exchange field. First-principles calculations of $G_{\uparrow \downarrow}^{r}$ and $G_{\uparrow \downarrow}^{t}$ have been carried out by Zwierzycki et al. ${ }^{27}$ showing small variation of the first and nonvanishing value of the second when the ferromagnetic layer becomes of the order of several monolayers.

\section{B. Observation of ferromagnetic coherence in transport experiments}

In this section we address coherence effects due to the transmission of transverse spin currents through ultrathin ferromagnetic layers or weak ferromagnets. These effects should be observable in Py structures when $d_{F} \lesssim 1.5 \mathrm{~nm}$. Band structure calculations show that in $\mathrm{Cu}|\mathrm{Co}| \mathrm{Cu}$ structures the mixing transmission can easily reach $G_{\uparrow \downarrow}^{t} \approx 0.1\left(G_{\uparrow}+G_{\downarrow}\right)$ for such thicknesses. ${ }^{27}$

We may draw an important conclusion from the extended magnetoelectronic circuit theory applied to general (asymmetric) $N 1|F 1| N 2|F 2| N 3$ structures: when the nodes are chosen in the middle normal metal and in the outer normal metals at the points that connect to the baths, a possibly finite-mixing transmission completely drops out of the charge transport equations, i.e., the expressions remain exactly the same as those presented above for the $N|F| N|F| N$ structure. For example, the charge and spin currents from $N 1$ (and similarly from $N 3$ ) into $N 2$ read

$$
\begin{aligned}
I_{0}= & \left(G_{\uparrow}^{N 1 \mid N 2}+G_{\downarrow}^{N 1 \mid N 2}\right) \Delta \mu_{0}^{N}+\left(G_{\uparrow}^{N 1 \mid N 2}-G_{\downarrow}^{N 1 \mid N 2}\right) \Delta \boldsymbol{\mu}_{s}^{N} \cdot \mathbf{m}_{\mathbf{z}}, \\
\mathbf{I}_{s}= & \mathbf{m}_{\mathbf{z}}\left[\left(G_{\uparrow}^{N 1 \mid N 2}-G_{\downarrow}^{N 1 \mid N 2}\right) \Delta \mu_{0}^{N}+\left(G_{\uparrow}^{N 1 \mid N 2}+G_{\downarrow}^{N 1 \mid N 2}\right) \Delta \boldsymbol{\mu}_{s}^{N}\right] \\
& -2\left(\mathbf{m}_{\mathbf{z}} \times \boldsymbol{\mu}_{s}^{N 2} \times \mathbf{m}_{\mathbf{z}}\right) \operatorname{Re} G_{\uparrow \downarrow}^{r N 2 \mid F 1} \\
& +2\left(\boldsymbol{\mu}_{s}^{N 2} \times \mathbf{m}_{\mathbf{z}}\right) \operatorname{Im} G_{\uparrow \downarrow}^{r N 2 \mid F 1} \\
& +2\left(\mathbf{m}_{\mathbf{z}} \times \boldsymbol{\mu}_{s}^{N 1} \times \mathbf{m}_{\mathbf{z}}\right) \operatorname{Re} G_{\uparrow \downarrow}^{t N 1 \mid N 2} \\
& -2\left(\boldsymbol{\mu}_{s}^{N 1} \times \mathbf{m}_{\mathbf{z}}\right) \operatorname{Im} G_{\uparrow \downarrow}^{t N 1 \mid N 2},
\end{aligned}
$$

where $\Delta \mu_{0}^{N}=\mu_{0}^{N 1}-\mu_{0}^{N 2}$ and $\Delta \boldsymbol{\mu}_{s}^{N}=\boldsymbol{\mu}_{s}^{N 1}-\boldsymbol{\mu}_{s}^{N 2}$ describe the potential and spin accumulation drops between the left and the middle nodes. By conservation of spin and charge currents in the center node, expression for aMR can be derived. However, the mixing transmission does not appear in Eqs. (13) and (14) since there is no spin accumulation in the outer 


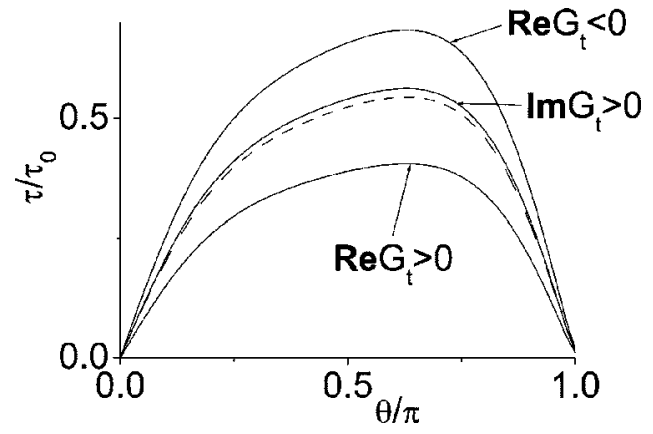

FIG. 8. The torque on the thin right layer of thickness $d=0.27 l_{s d}$ for $F|N| F$ structure. The left layer has thickness $d \gg l_{s d}^{F}$. The curves starting from the lower one respectively, $\operatorname{Re}\left(1 / G_{\uparrow \downarrow}^{t}\right)$ $=5 \mathrm{f} \Omega \mathrm{m}^{2}, \quad \operatorname{Im}\left(1 / G_{\uparrow \downarrow}^{t}\right)=\infty ; \operatorname{Re}\left(1 / G_{\uparrow \mid}^{t}\right)=\infty, \operatorname{Im}\left(1 / G_{\uparrow \downarrow}^{t}\right)=5 \mathrm{f} \Omega \mathrm{m}^{2} ;$ $\operatorname{Re}\left(1 / G_{\uparrow \downarrow}^{t}\right)=\infty, \operatorname{Im}\left(1 / G_{\uparrow \downarrow}^{t}\right)=\infty ; \operatorname{Re}\left(1 / G_{\uparrow \downarrow}^{t}\right)=-5 \mathrm{f} \Omega \mathrm{m}^{2}, \operatorname{Im}\left(1 / G_{\uparrow \downarrow}^{t}\right)$ $=\infty\left(\tau_{0}=I_{0} \hbar / 2 e\right)$.

nodes (reservoirs). Ferromagnets thin enough to allow transmission of a transverse spin current can therefore not be distinguished from conventional ones in the aMR. Our conclusions therefore disagree with the claims of ferromagnetic coherence effects in aMR experiments on $N|F| N|F| N$ structures by Urazhdin et $a .^{23}$

On the other hand, the torque on the thin ferromagnet $F 2$ does change as follows:

$$
\begin{aligned}
\vec{\tau}_{2}= & -2\left(\mathbf{m}_{\mathbf{z}} \times \boldsymbol{\mu}_{s}^{N 2} \times \mathbf{m}_{\mathbf{z}}\right) \operatorname{Re}\left(G_{\uparrow \downarrow}^{r N 2 \mid F 2}-G_{\uparrow \downarrow}^{t N 2 \mid N 3}\right) \\
& +2\left(\boldsymbol{\mu}_{s}^{N 2} \times \mathbf{m}_{\mathbf{z}}\right) \operatorname{Im}\left(G_{\uparrow \downarrow}^{r N 2 \mid F 2}-G_{\uparrow \downarrow}^{t N 2 \mid N 3}\right) .
\end{aligned}
$$

A parametrization of the torque via a combination $G_{\uparrow \downarrow}^{r N 2 \mid F 2}-G_{\uparrow \downarrow}^{t N 2 \mid N 3}$ was found in Ref. 13 by random matrix theory, which is equivalent with circuit theory when the number of transverse channels is large. ${ }^{22}$ However, these authors did not discuss their results in the limit of thin ferromagnetic layers. When $\operatorname{Im} G_{\uparrow \downarrow}^{t} \approx 0$ and $\operatorname{Im} G_{\uparrow \downarrow}^{r} \approx 0$, the torque $\tau_{\text {coh }}$ acting on the thin layer is modified from the incoherent expression $\tau$ as

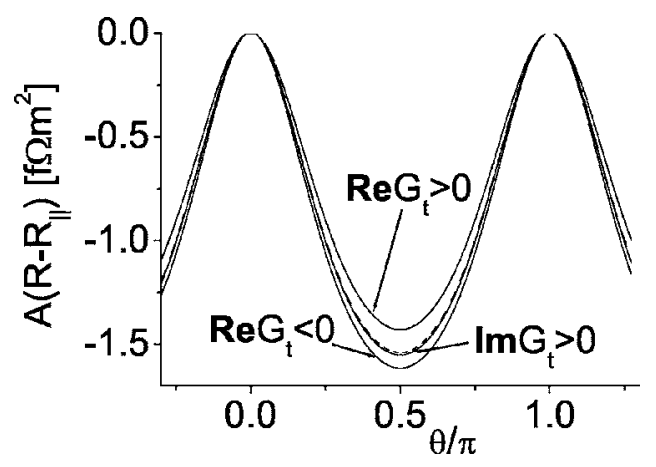

FIG. 9. aMR of $F|N| F|N| F$ structure for the thickness of the middle layer $d=0.27 l_{s d}$. Outer layers are antiparallel with $d \gg l_{s d}^{F}$. The curves starting from the lower one respectively, $\operatorname{Re}\left(1 / G_{\uparrow \mid}^{t}\right)=-5 \mathrm{f} \Omega \mathrm{m}^{2}, \operatorname{Im}\left(1 / G_{\uparrow \mid}^{t}\right)=\infty ; \operatorname{Re}\left(1 / G_{\uparrow \mid}^{t}\right)=\infty, \operatorname{Im}\left(1 / G_{\uparrow \mid}^{t}\right)$ $=5 \mathrm{f} \Omega \mathrm{m}^{2} ; \operatorname{Re}\left(1 / G_{\uparrow \downarrow}^{t}\right)=\infty, \quad \operatorname{Im}\left(1 / G_{\uparrow \downarrow}^{t}\right)=\infty ; \operatorname{Re}\left(1 / G_{\uparrow \downarrow}^{t}\right)=5 \mathrm{f} \Omega \mathrm{m}^{2}$, $\operatorname{Im}\left(1 / G_{\uparrow \downarrow}^{t}\right)=\infty$.

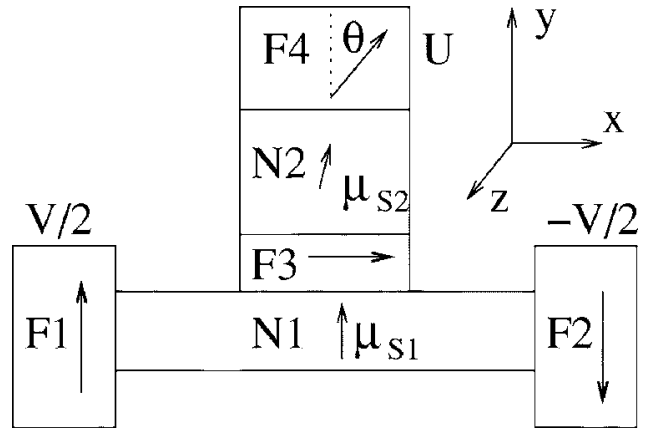

FIG. 10. An experimental setup to observe the mixing transmission and measure the ferromagnetic coherence length in ferromagnet $F 3$. The spin accumulation $\vec{\mu}_{S 2}$ in the normal metal $N 2$ is measured via the angular dependence of the potential $U(\theta)$ of the ferromagnet $F 4$ that is weakly coupled to $N 2$.

$$
\tau_{\mathrm{coh}}=\tau\left(G_{\uparrow \downarrow}^{r}-G_{\uparrow \downarrow}^{t}\right) / G_{\uparrow \downarrow}^{r} .
$$

Naively one may expect that the reduced absorption of the transverse spin accumulation diminishes the torque, but this is not necessarily so (see Fig. 8). Since the mixing transmission may be negative, Eq. (15) shows that increased torques are possible. This can be understood as follows. A spin entering a ferromagnet will precess around an exchange field normal to its quantization axis. A negative mixing transmission $\operatorname{Re} G_{\uparrow \downarrow}^{t}<0$ adds a phase factor corresponding to a rotation over an angle $\pi$ during transmission. The outgoing spin then has a polarization opposite to the incoming one. The magnetization torque, i.e., the difference between in and outgoing spin currents, consequently increases compared to the situation in which the incoming transverse spin is absorbed as in thick ferromagnetic layers.

In contrast to $N|F| N|F| N$ structures, we find that it $i s$ possible to observe $G_{\uparrow \downarrow}^{t}$ in the aMR of $F|N| F|N| F$ devices. We study here the dependence of the aMR on the mixing transmission in a Py-based multilayer. In Fig. 9 we present the aMR for different mixing transmissions in the middle layer of thickness $d_{F}=0.27 l_{s d}$. Unfortunately, it seems difficult to obtain quantitative values for the mixing transmission from experiments since the dependence of the aMR on $G_{\uparrow \downarrow}^{t}$ is rather weak.

When the coherence length becomes larger than the scattering mean-free path, which can occur in weak ferromagnets like $\mathrm{PdNi}$ or $\mathrm{CuNi}$, the transverse spin accumulation should be treated by a diffusion equation. ${ }^{36}$ The result can be parametrized again in terms of a mixing transmission, which can subsequently be used in our circuit theory.

\section{Three-terminal device for observation of coherence effects}

Finally, we propose an experiment that should be more sensitive to ferromagnetic coherence. We suggest the setup shown in Fig. 10 that is analogous to the spin-torque transistor $^{37}$ and the magnetoelectronic spin-echo ${ }^{32}$ concepts. A current through the antiparallel ferromagnets $F 1$ and $F 2$ excites a spin accumulation in the normal metal $N 1$. This spin accumulation can transmit $F 3$ only when its thickness is less than $\lambda_{c}$. In that case a spin accumulation is induced in 
the upper normal metal $N 2$ that can be detected as a voltage depending on the magnetization angle $\theta$ of the analyzing ferromagnet $F 4$. We assume here that $N 1$ is smaller than its spin-flip diffusion length $(\mathrm{Cu}$ is a good candidate with spindiffusion lengths of up to a micron) such that the spin accumulation is constant under the contact to $F 3$. Otherwise the signal at the ferromagnet $F 4$ is diminished since part of the spin accumulation in $N 1$ is lost due to spin-flip processes.

When the $G_{\uparrow \downarrow}^{t}$ of $F 3$ is smaller than its $G_{\uparrow}^{r}$ and ferromagnet $F 4$ is not too leaky for the spin current (e.g., connected via a tunnel junction) the spin accumulation in $N 2$ can be found from Eqs. (10) and (11) in terms of the spin accumulation in $N 1$,

$$
\begin{aligned}
\boldsymbol{\mu}_{S 2}= & \frac{\left|\mu_{S 1}\right|}{\left(\operatorname{Re} G_{\uparrow \downarrow}^{r}\right)^{2}+\left(\operatorname{Im} G_{\uparrow \downarrow}^{r}\right)^{2}} \\
& \times\left(\begin{array}{c}
0 \\
\operatorname{Re} G_{\uparrow \downarrow}^{r} \operatorname{Re} G_{\uparrow \downarrow}^{t}+\operatorname{Im} G_{\uparrow \downarrow}^{r} \operatorname{Im} G_{\uparrow \downarrow}^{t} \\
\operatorname{Re} G_{\uparrow \downarrow}^{r} \operatorname{Im} G_{\uparrow \downarrow}^{t}-\operatorname{Im} G_{\uparrow \downarrow}^{r} \operatorname{Re} G_{\uparrow \downarrow}^{t}
\end{array}\right) \\
& \stackrel{\operatorname{Im} G_{\uparrow \downarrow}^{r} \rightarrow 0}{\simeq} \frac{\left|\mu_{S 1}\right|}{\operatorname{Re} G_{\uparrow \downarrow}^{r}}\left(\begin{array}{c}
0 \\
\operatorname{Re} G_{\uparrow \downarrow}^{t} \\
\operatorname{Im} G_{\uparrow \downarrow}^{t}
\end{array}\right),
\end{aligned}
$$

where Eq. (17) holds to a good approximation when the layer $F 3$ is metallic. The spin accumulation is indeed coherently rotated by the exchange field in $F 3$. The angle dependence of the potential in $F 4$ is $U(\theta) \approx \mu_{S 1} P\left|G_{\uparrow \downarrow}^{t}\right| \cos \theta /\left(\operatorname{Re} G_{\uparrow \downarrow}^{r}\right)$ with maximum along $\boldsymbol{\mu}_{S 2}$, where $P$ is the polarization of the contact $N 2 \mid F 4$.

When the $G_{\uparrow \downarrow}^{t}$ of $F 3$ is not smaller than $G_{\uparrow \downarrow}^{r}$ (or the spin current leak into $F 4$ is significant), the spin accumulation $\boldsymbol{\mu}_{S 1}$ is affected by $\boldsymbol{\mu}_{S 2}$ and the final expressions are more complicated.

An angle dependence of $U(\theta)$ provides a direct proof of a finite-mixing transmission. The ferromagnetic coherence length can be determined by repeating experiments for a number of layer thicknesses of $F 3$. Such a direct experimental evidence should help to get a grip on this important parameter $\lambda_{c}$.

\section{CONCLUSION}

In this paper we extracted the spin-mixing conductance of a Py $\mid \mathrm{Cu}$ interface from the experimental data of Urazhdin et al. using material parameters measured independently by the MSU collaboration. ${ }^{34}$ We find good agreement with experiments on asymmetric $F|N| F \mid N$ multilayers, reproducing quantitatively the nonmonotonic aMR that we predicted earlier. ${ }^{24-26}$ Magnetoelectronic circuit theory together with the diffusion equation is a convenient tool for the data analysis when the spin-flip diffusion length in the ferromagnet is of the same order as the layer thickness. We suggest carrying out current-induced magnetization reversal experiments on samples that display the nonmonotonic aMR since we predict anomalous magnetization trajectories due to a vanishing torque at finite magnetization angle. ${ }^{24-26}$ We also study the effects of the finite ferromagnetic coherence length in ultrathin ferromagnetic films or weak ferromagnets. For this purpose the magnetoelectronic circuit theory is extended to treat phase coherent transport in the ferromagnet. A coherence length that is larger than the ferromagnetic layer thickness does not modify the aMR of $N|F| N|F| N$ structures, but a small effect should exist in $F|N| F|N| F$ structures. In contrast, the spin-transfer torque is affected more strongly and may even be increased by the spin-coherence when the exchange field rotates the transverse spin-current polarization by the angle $\pi$. Finally, we propose a three-terminal device that should allow experimental determination of the ferromagnetic coherence length.

We thank Yaroslav Tserkovnyak for helpful discussions. This work has been supported by the Dutch FOM Foundation and the Research Council of Norway, the EU Commission FP6 NMP-3 project 505587-1 "SFINX", NANOMAT Grants No. 158518/143 and 158547/431.

\section{APPENDIX: ANALYTICAL RESULTS FOR $F|N| F|N| F$ STRUCTURE}

The aMR of a $F(\uparrow)|N| F(\theta)|N| F(\uparrow / \downarrow)$ CPP pillar can be described analytically when the thick outer layers are parallel or antiparallel, respectively.

$$
\begin{gathered}
R(\theta)=2\left(R_{\uparrow \downarrow}+R\right)+R_{M}-\frac{R_{\uparrow \downarrow}\left(R_{M-}^{2}+4 R_{-}\left(R_{-}+R_{M-} \alpha\right)\right)+\left(2 R_{M} R_{-}^{2}+R_{M-}^{2} R\right)\left(1-\alpha^{2}\right)}{\left(R_{\uparrow \downarrow}+R\right)\left(2 R_{\uparrow \downarrow}+R_{M}\right)-R R_{M} \alpha^{2}}, \\
R(\theta)=2\left(R_{\uparrow \downarrow}+R\right)+R_{M}-\frac{2 R_{-}^{2}\left(1-\alpha^{2}\right)}{R_{\uparrow \downarrow}+R\left(1-\alpha^{2}\right)}-\frac{2 R_{M-}^{2}\left(R_{\uparrow \downarrow}+R\left(1-\alpha^{2}\right)\right)}{\left(R_{\uparrow \downarrow}+R\right)\left(2 R_{\uparrow \downarrow}+R_{M}\right)-R R_{M} \alpha^{2}},
\end{gathered}
$$

where $\alpha=\cos \theta, 4 R+2 R_{\uparrow \downarrow}=1 / G_{\uparrow}+1 / G_{\downarrow}, 4 R_{-}=1 / G_{\uparrow}-1 / G_{\downarrow}$ for the outer layers. The mixing resistance for two interfaces adjacent to any normal metal $R_{\uparrow \downarrow}=1 / G_{\uparrow \downarrow}^{r}$ (we assume all interfaces identical). For the middle layer $4 R_{M}=1 / G_{\uparrow}+1 / G_{\downarrow}$ $-4 R_{\uparrow \downarrow}, 4 R_{M-}=1 / G_{\uparrow}-1 / G_{\downarrow}$. 
${ }^{1}$ M. N. Baibich, J. M. Broto, A. Fert, F. Nguyen Van Dau, F. Petroff, P. Eitenne, G. Creuzet, A. Friederich, and J. Chazelas, Phys. Rev. Lett. 61, 2472 (1988).

${ }^{2}$ M. A. M. Gijs and G. E. W. Bauer, Adv. Phys. 46, 285 (1997).

${ }^{3}$ J. C. Slonczewski, J. Magn. Magn. Mater. 159, L1 (1996).

${ }^{4}$ L. Berger, Phys. Rev. B 54, 9353 (1996).

${ }^{5}$ E. B. Myers, D. C. Ralph, J. A. Katine, R. N. Louie, and R. A. Buhrman, Science 285, 867 (1999).

${ }^{6}$ M. Tsoi, A. G. M. Jansen, J. Bass, W. C. Chiang, M. Seck, V. Tsoi, and P. Wyder, Phys. Rev. Lett. 80, 4281 (1998).

${ }^{7}$ M. Tsoi, A. Jansen, J. Bass, W. Chiang, M. Seck, V. Tsoi, and P. Wyder, Phys. Rev. Lett. 81, 493 (1998).

${ }^{8}$ J. Wegrowe, D. Kelly, Y. Jaccard, P. Guittienne, and J. Ansermet, Europhys. Lett. 45, 626 (1999).

${ }^{9}$ I. N. Krivorotov, N. C. Emley, J. C. Sankey, S. I. Kiselev, D. C. Ralph, and R. A. Buhrman, Science 307, 228 (2005).

${ }^{10}$ T. Valet and A. Fert, Phys. Rev. B 48, 7099 (1993).

${ }^{11}$ J. Bass and W. Pratt, Jr., J. Magn. Magn. Mater. 200, 274 (1999).

${ }^{12}$ C. Galinon, K. Tewolde, R. Loloee, W.-C. Chiang, S. Olson, H. Kurt, W. Pratt, Jr., J. Bass, P. Xu, K. Xia, and M. Talanana (unpublished).

${ }^{13}$ X. Waintal, E. B. Myers, P. W. Brouwer, and D. C. Ralph, Phys. Rev. B 62, 12317 (2000).

${ }^{14}$ A. Brataas, Y. V. Nazarov, and G. E. W. Bauer, Eur. Phys. J. B 22, 99 (2001).

15 J. C. Slonczewski, J. Magn. Magn. Mater. 247, 324 (2002).

${ }^{16}$ M. D. Stiles and A. Zangwill, Phys. Rev. B 66, 014407 (2002).

${ }^{17}$ A. Brataas, Y. V. Nazarov, and G. E. W. Bauer, Phys. Rev. Lett. 84, 2481 (2000).

${ }^{18}$ K. Xia, P. J. Kelly, G. E. W. Bauer, A. Brataas, and I. Turek, Phys. Rev. B 65, 220401(R) (2002).

${ }^{19}$ P. Dauguet, P. Gandit, J. Chaussy, S. F. Lee, A. Fert, and P. Holody, Phys. Rev. B 54, 1083 (1996).

${ }^{20}$ A. Vedyayev, N. Ryzhanova, B. Dieny, P. Dauguet, P. Gandit, and J. Chaussy, Phys. Rev. B 55, 3728 (1997).
${ }^{21}$ L. Giacomoni, B. Dieny, W. Pratt, Jr., R. Loloee, and M. Tsoi (unpublished).

${ }^{22}$ G. E. W. Bauer, Y. Tserkovnyak, D. Huertas-Hernando, and A. Brataas, Phys. Rev. B 67, 094421 (2003).

${ }^{23}$ S. Urazhdin, R. Loloee, and W. P. Pratt, Jr., Phys. Rev. B 71, 100401(R) (2005).

${ }^{24}$ A. A. Kovalev, A. Brataas, and G. E. W. Bauer, Phys. Rev. B 66, 224424 (2002).

${ }^{25}$ J. Manschot, A. Brataas, and G. E. W. Bauer, Phys. Rev. B 69, 092407 (2004).

${ }^{26}$ J. Manschot, A. Brataas, and G. Bauer, Appl. Phys. Lett. 85, 3250 (2004).

${ }^{27}$ M. Zwierzycki, Y. Tserkovnyak, P. J. Kelly, A. Brataas, and G. E. W. Bauer, Phys. Rev. B 71, 064420 (2005).

${ }^{28}$ A. Shpiro, P. M. Levy, and S. Zhang, Phys. Rev. B 67, 104430 (2003).

${ }^{29}$ L. Berger, J. Appl. Phys. 93, 7693 (2003).

${ }^{30}$ S. Nakamura, J. Min. Mater. Process. Inst. Jpn. 43, 498 (2004).

${ }^{31}$ Y. Tserkovnyak, A. Brataas, and G. E. W. Bauer, Phys. Rev. Lett. 88, 117601 (2002).

${ }^{32}$ A. Brataas, G. Zarand, Y. Tserkovnyak, and G. E. W. Bauer, Phys. Rev. Lett. 91, 166601 (2003).

${ }^{33}$ J. Xiao, A. Zangwill, and M. D. Stiles, Phys. Rev. B 70, 172405 (2004).

${ }^{34}$ W. Pratt, Jr. S. D. Steenwyck, S. Y. Hsu, W-C. Chiang, A. C. Schaefer, R. Loloee, and J. Bass, IEEE Trans. Magn. 33, 3505 (1997).

${ }^{35}$ S. Urazhdin, R. Loloee, and W. Pratt, Jr., cond-mat/0403441 v1 (unpublished).

${ }^{36}$ S. Zhang, P. M. Levy, and A. Fert, Phys. Rev. Lett. 88, 236601 (2002).

${ }^{37}$ G. E. W. Bauer, A. Brataas, Y. Tserkovnyak, and B. J. van Wees, Appl. Phys. Lett. 82, 3928 (2003).

${ }^{38} \mathrm{~S}$. Nakamura et al. (unpublished). 